\title{
A multiscale model for the ductile fracture of crystalline materials
}

\author{
Yueguang Wei ${ }^{\mathrm{a}, *}$, Guanshui Xu ${ }^{\mathrm{b}}$ \\ ${ }^{a}$ LNM, Institute of Mechanics, Chinese Academy of Sciences, Beijing 100080, PR China \\ ${ }^{\mathrm{b}}$ Department of Mechanical Engineering, University of California at Riverside, Riverside, CA 92521, USA
}

Received 6 February 2004

Available online 27 June 2005

\begin{abstract}
In this paper, a multiscale model that combines both macroscopic and microscopic analyses is presented for describing the ductile fracture process of crystalline materials. In the macroscopic fracture analysis, the recently developed strain gradient plasticity theory is used to describe the fracture toughness, the shielding effects of plastic deformation on the crack growth, and the crack tip field through the use of an elastic core model. The crack tip field resulting from the macroscopic analysis using the strain gradient plasticity theory displayes the $1 / 2$ singularity of stress within the strain gradient dominated region. In the microscopic fracture analysis, the discrete dislocation theory is used to describe the shielding effects of discrete dislocations on the crack growth. The result of the macroscopic analysis near the crack tip, i.e. a new $\mathrm{K}$-field, is taken as the boundary condition for the microscopic fracture analysis. The equilibrium locations of the discrete dislocations around the crack and the shielding effects of the discrete dislocations on the crack growth at the microscale are calculated. The macroscopic fracture analysis and the microscopic fracture analysis are connected based on the elastic core model. Through a comparison of the shielding effects from plastic deformation and the discrete dislocations, the elastic core size is determined.
\end{abstract}

(C) 2005 Elsevier Ltd. All rights reserved.

Keywords: Discrete dislocation theory; Strain gradient plasticity theory; Bridging model; Macro/microscopic fracture process; Energy release rate

\footnotetext{
${ }^{*}$ Corresponding author. Tel.: +86 1062648721 ; fax: +86 1062561284.

E-mail address: ywei@lnm.imech.ac.cn (Y. Wei).
} 


\section{Introduction}

The fracture behavior of a structured material is governed by processes occurring over a diverse range of length scales. Most models of the fracture processes, however, usually focus on the process over a limited range of length scales. Continuum models based on elastic, elastic-plastic, and recently developed strain gradient constitutive relations are appropriate for the analysis of the macro/microscopic mechanical response of solids, but they do not accurately represent the underlying fundamental deformation mechanism of crystal defects such as nucleation and motion of discrete dislocations and slip of grain boundaries. On the other hand, the discrete/continuum models, such as the discrete dislocation theory, may be capable of accounting for the fundamental dislocation mechanisms in the deformation and fracture process, but at the present stage of the development, this kind of model is limited to the consideration of nominally elastic behavior (i.e. relatively small or non-existent dislocation densities) at very small scale. The multiple-scale approaches toward materials modeling have led to a wealth of understanding of materials mechanical behavior within each domain of the model's applicability. However, it is still of central importance to address the linkage between the models at the different length scales in order to develop mechanism based modeling of mechanical behavior of materials.

In this paper, we develop a multiscale model that builds upon the recently developed elastic-plastic and strain gradient models for plastic deformation at the macroscale and the discrete dislocation for plastic deformation at the submicron scale. In our model, we attempt to establish the linkage between these two models to address the large disparity between the relevant length-scales involved in the ductile fracture processes at the crack tip.

Ductile fracture processes have been modeled extensively based on both continuum mechanics and discrete dislocation dynamics theories. In continuum mechanics-based models, stress fields of elastic-plastic crack tips have been solved based on the conventional elastic-plastic theory by using asymptotic methods and finite element methods, and fracture criteria have been developed (Betegon and Hancock, 1991; O’Dowd and Shih, 1991; Xia et al., 1993; Tvergaard and Hutchinson, 1993; Wei and Wang, 1995a,b; Khan and Khraisheh, 2004). These results indicate that during crack growth the crack surface separation strength near the crack tip is about 4-6 times the yield strength of the material. This strength, however, is relatively lower and not consistent with the result from microscopic fracture analyses (Hong et al., 1994; Raynolds et al., 1996; Evans et al., 1999). Recently, elastic-plastic crack tip fields which reflect strain gradient effects have been presented. The results show that the separation strength of near-tip crack surface undergoes a considerable increase (Wei and Hutchinson, 1997a; Jiang et al., 2001; Chen and Wang, 2002). This seems to set up a hope to link the macroscopic fracture analysis to the microscopic fracture analysis. However, a question still remains: what is the lower limit beyond which the strain gradient theory will not be suitable? On the other hand, at the atomistic scale, the competing mechanisms between cleavage crack growth and dislocation emission have been studied considerably (Rice and Thomson, 1974; Lin and Thomson, 1986; Rice, 1992; Xu and Argon, 1995; 1997; Wang, 1998; Yang et al., 2001). Furthermore, 
the brittle-ductile transition and crack growth considering the discrete dislocation shielding effect have been investigated (Hsia et al., 1994; Giessen and Needleman, 1995; Mao and Evans, 1997; Xin and Hsia, 1997; Mao and Li, 1999; Argon, 2001). Although these models reveal the local physical mechanisms of fracture processes, they cannot practically incorporate the large scale plastic deformation surrounding the crack tip to precisely predict the macroscopic mechanical behavior of materials. The elastic-plastic continuum theory and discrete dislocation theory are obviously two different methodologies in simulating the shielding effects on the crack growth. One is built upon the micron-scale analysis, the other one is built upon the macro-scale analysis. Additionally, some bridging models related to the detailed fracture process characterizations at small scales have been presented (Needleman, 1987; Suo et al., 1993; Beltz et al., 1996; Lipkin et al., 1996; Wei and Hutchinson, 1999), and related to the ductile damage of polycrystalline materials have been presented more recently (Bonfoh et al., 2004). Specifically, the model of the elastic core zone (dislocation-free zone) near a growing crack tip has been defined and presented by Suo, Shih and Varias (1993, usually called the SSV model) and the zone size has been estimated by Beltz et al. (1996) from the dislocation point of view. In the present research, we attempt to develop a macro-/micro-scale bridging model for describing a complete fracture process accounting for the shielding effects, i.e., at the small scale, the discrete dislocation theory is used to model the shielding effect of discrete dislocations on the crack growth, while at the large scale, the continuum theory (strain gradient plasticity theory) is adopted to model the shielding effect of plastic deformation on the crack growth. We establish the condition to link these two models.

\section{Model descriptions}

A linkage model between the macroscopic and the microscopic fracture analysis is presented, as shown in Fig. 1. The entire description of a material fracture process should consist of both the macroscopic fracture process and the microscopic fracture process. These fracture characteristics can be described by using the continuum

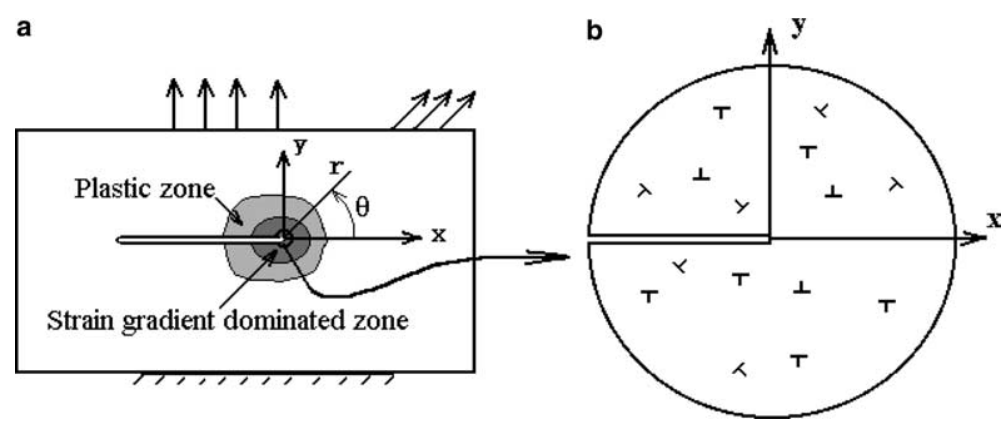

Fig. 1. The sketch of the macro-/micro-scale bridging model. 
model and the discrete dislocation model, respectively, as sketched in Figs. 1(a) and (b). In Fig. 1(a), the material fracture behavior within the micron-scale level which is larger than a micron can be predicted by using the conventional continuum model, i.e., the conventional elastic-plastic theory and recently developed strain gradient theory. In this scale, there exist three regions around the crack tip with loading: the elastic zone far away from the tip, the strain gradient dominated zone very near the crack tip and the plastic zone between the elastic zone and the strain gradient zone. Actually, within a small scale which is smaller than a micron, the material fracture behavior can be predicted by using the discrete dislocation theory, as shown in Fig. 1(b). With increasing load, dislocations nucleate and emit from the crack tip, and discrete dislocations exist (in equilibrium) within the region. The key problems noted here are: (1) what is the discrete dislocation number in equilibrium or in limit equilibrium for a given material under a given loading condition? (2) what is the effective size of the discrete dislocation region? and (3) in addition, what are the macro/micro-scale linkage conditions, i.e., the outer boundary conditions of the discrete dislocation model, or the inner boundary conditions of the conventional continuum model? In the present study, the macroscopic analysis results will be taken as the outer boundary conditions for the microscopic problem. The key point is to determine the intersection radius between the microscopic problem and the macroscopic problem. We shall study the problems in the present research.

\section{Fracture analyses using the continuum model}

For simplicity, a case of a semi-infinite length crack growing under steady-state, plane strain and the mode I loading conditions will be considered. For describing the multi-scale problem clearly, an elastic core model, or a modified SSV model is adopted here, as shown in Fig. 2. This model is an improvement over the conventional SSV model (Suo et al., 1993), which assumes that near the growing crack tip and the crack surface a dislocation-free zone strip with infinite length exists.

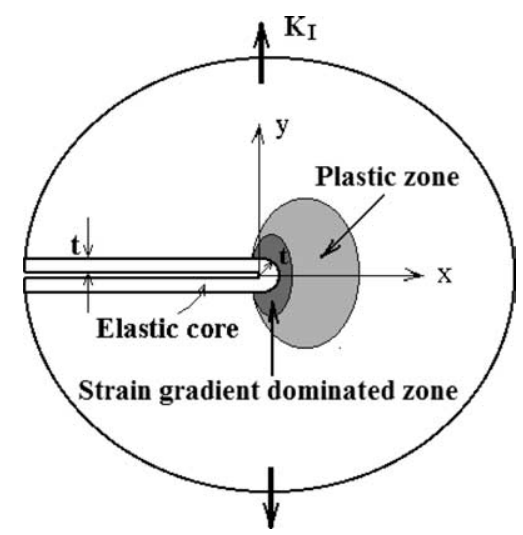

Fig. 2. Elastic core model for mode I crack case. 
Suo et al. (1993) noted that a sharp crack could grow slowly by cleavage along a gold/sapphire interface (Reimanis et al., 1991), a copper/sapphire interface (Beltz and Wang, 1992) as well as a niobium/alumina interface (O'Dowd et al., 1992), etc. They presented an elastic core model for crack growth within a ductile material or along a metal/ceramic interface based on the mechanism of dislocation emission and motion. According to the model, a growing crack tip is always kept in an elastic region. Based on the elastic core model (or the SSV model), Beltz et al. (1996); Wei and Hutchinson $(1997,1999)$ and Tvergaard (1997) studied the crack growth within a ductile materials or along an interface of metal/ceramic. In the present research, we use a modified SSV model to describe crack growth process in macroscopic scale. Under steady-state growth conditions, a semi-infinite elastic strip around the crack surface is left behind the crack tip. The radius of the elastic core (or thickness of the elastic layer), $t$, can be taken as a model parameter (referring to Wei and Hutchinson, 1997b, 1999).

The modified SSV model and the mechanism-based strain gradient (MSG) plasticity flow theory (Gao et al., 1999; Huang et al., 2000; see Appendix A) allow the relations of the normalized total energy release rate with the material parameters and the model parameter under steady-state advancement of the crack tip to be obtained through a dimensional analysis as,

$$
\frac{G_{\mathrm{ss}}}{G_{0}^{\prime}}=f\left(\frac{E}{\sigma_{\mathrm{Y}}}, v, N, \frac{l}{R_{0}}, \frac{R_{0}}{t}\right),
$$

where the length parameter $l$ describes the strain gradient effect, $t$ is the elastic core size, or intersection radius of the microscopic and macroscopic fields which is to be determined. The total energy release rate $G_{\mathrm{ss}}$ and the length parameter $R_{0}$ are defined as follows:

$$
G_{\mathrm{ss}}=\frac{K_{\mathrm{I}}^{2}\left(1-v^{2}\right)}{E}, \quad R_{0}=\frac{E G_{0}^{\prime}}{3 \pi\left(1-v^{2}\right) \sigma_{\mathrm{Y}}^{2}},
$$

where $K_{\mathrm{I}}$ is the stress intensity factor (applied external load). $R_{0}$ is the plastic zone size in small scale yielding. $G_{0}^{\prime}$ is the macroscopic fracture toughness, or the crack surface separation energy. Through finite element numerical simulation by using the MSG strain gradient theory, the details of the parameter relation in (1) are given in Fig. 3 for several parameter values. The numerical process is similar to that by Wei et al. (2004). Fig. 3 shows that the variation of the normalized energy release rate is very sensitive to the elastic core size and length parameter $R_{0}$. For the typical metallic materials, $E / \sigma_{\mathrm{Y}} \approx 500, G_{0}^{\prime}=1$ to $4 \mathrm{~J} \mathrm{~m}^{-2}, \sigma_{\mathrm{Y}}=200 \mathrm{MPa}$ and $v=0.3$, the value of $R_{0}$ is about one micron.

Therefore, when the elastic core size of the macroscopic model is taken to be submicron, the normalized energy release rate is quite sensitive to the value of the elastic core size.

In order to determine the remote boundary condition for microscopic fracture analysis later, we need to investigate the characteristics of the stress field singularity for macroscopic fracture problem. For this reason, the effective stress distribution 


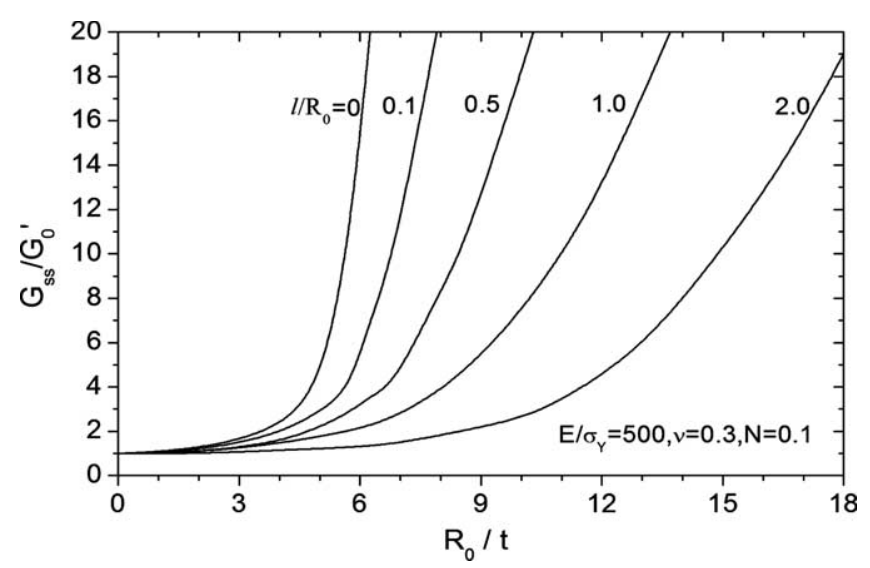

Fig. 3. Macroscopic fracture solutions of the normalized energy release rate based on the strain gradient theory and the elastic core model ( $t$ is elastic core thickness).

near the crack tip under the action of remote $K_{\mathrm{I}}$ field (considering the mode I case) will be calculated, and the normalized relation can be dictated as by dimensional analysis to be

$$
\frac{\sigma_{\mathrm{e}}}{\sigma_{\mathrm{Y}}}=g\left(\frac{R_{\mathrm{P}}}{r} ; \frac{E}{\sigma_{\mathrm{Y}}}, v, N, \frac{l}{R_{\mathrm{P}}}\right),
$$

where $R_{\mathrm{P}}$ is defined as

$$
R_{\mathrm{P}}=\frac{K_{\mathrm{I}}^{2}}{3 \pi \sigma_{\mathrm{Y}}^{2}}=\frac{E G_{\mathrm{ss}}}{3 \pi\left(1-v^{2}\right) \sigma_{\mathrm{Y}}^{2}} .
$$

Figs. 4(a) and (b) show the variations of the effective stress ahead of crack tip. In the figures, the relation of normalized effective stress with the distance away from crack tip is plotted on a logarithmic scale for easy checking of the singularity. The results shown in Figs. 4(a) and (b) correspond to the case $N=0.1$ and the case $N=0.2$, respectively. In Fig. 4, for small values of $R_{\mathrm{P}} / r$ (corresponding to small external loading (small $K_{\mathrm{I}}$ ) or large $r$ (the location far away from the crack tip)), conventional (1/2) singularity is obvious. For large values of $R_{\mathrm{P}} / r$ (corresponding to large external loading exerted or small $r$ (the location very near the crack tip)), the material undergoes plastic deformation. For the conventional elastic-plastic case $\left(\ell / R_{\mathrm{P}}=0.0\right)$, in the plastic zone the effective stress has the $\left(1 / 1+N^{-1}\right)$ singularity (HRR field). As we further approach the crack tip, the strain gradient effect prevails as shown in Fig. 4 and the stress singularity appears approximately to be (1/2) again as in the elastic case. However, within the strain gradient zone the corresponding stress intensity factor $K^{\prime}=\sqrt{E G_{0}^{\prime} /\left(1-v^{2}\right)}$ is considerably smaller than that acting on the remote boundary, $K=\sqrt{E G_{\mathrm{ss}} /\left(1-v^{2}\right)}$, due to the fact that $G_{0}^{\prime} \ll G_{\text {ss }}$, especially for small value of $l / R_{\mathrm{P}}$, as shown in Fig. 4(b). The value of $K / K^{\prime}$ corresponds to the ratio of effective stresses at point 1 and at point 2, as shown in Fig. 4. For a stationary crack, there are similar characteristics (Jiang et al., 2001; Chen and Wang, 


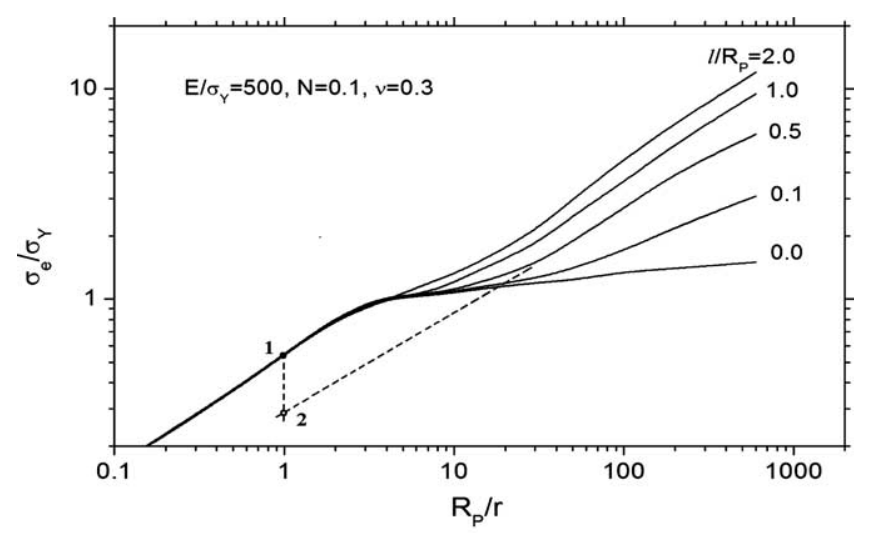

(a) $\mathrm{N}=0.1$

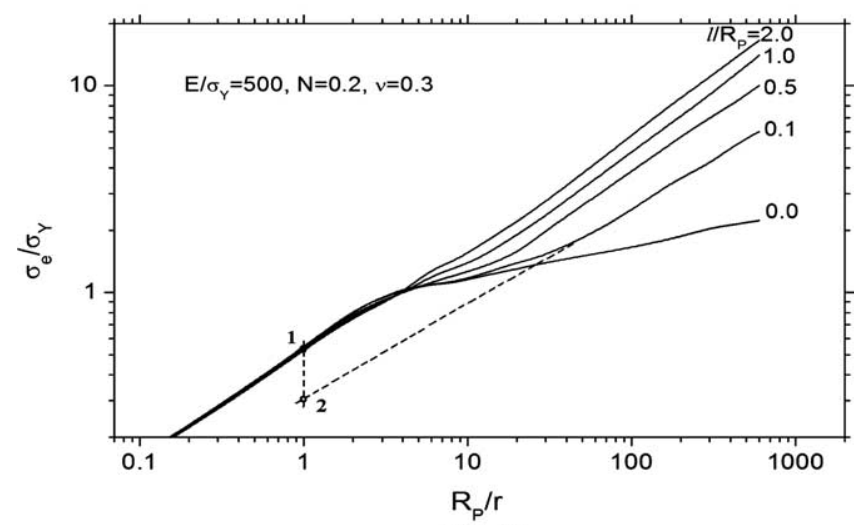

(b) $\mathrm{N}=0.2$

Fig. 4. Effective stress distribution near growing crack tip based on the strain gradient theory. Within the strain gradient dominated zone, solution behaves the $1 / 2$ singularity.

2002), as shown in Fig. 5; and the results shown in Fig. 5 come from Jiang et al. (2001) for different horizontal coordinates. This implies that the boundary condition of the microscopic model (see Fig. 1(b)) can be approximately taken as a $K^{\prime}$-field (we refer to it as $K^{\prime}$-field in the present paper for the purpose of distinguishing it from the macroscopic case in Fig. 3). Thus, the linkage conditions will include the stress level from Figs. 4 and 5 and the following relation:

$$
G_{0}^{\prime}=\frac{K_{\mathrm{I}}^{\prime 2}\left(1-v^{2}\right)}{E} .
$$

It is worth noting that in the analysis of a crack problem for a ductile material using the discrete dislocation theory, if a $K^{\prime}$-field acting on the boundary is considered, actually the $K^{\prime}$-field strength (stress intensity factor) must be much smaller than that exerted on the remote boundary. 


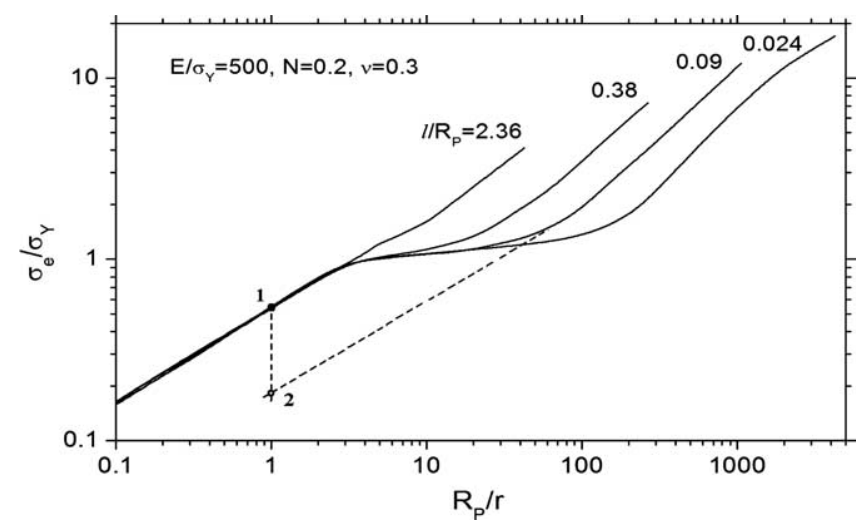

Fig. 5. Effective stress distribution near stationary crack tip based on the strain gradient theory. Obviously, within the strain gradient dominated zone, solution behaves the $1 / 2$ singularity. From Jiang et al. (2001).

\section{Fracture analyses using the discrete dislocation model}

As discussed above, the macroscopic fracture process is accompanied by a microscopic fracture process within the submicron zone near the crack tip. For the microscopic fracture analysis, the discrete dislocation theory is adopted here. A simplified presentation of the model has been given previously, in Fig. 1(b). In order to present the problem completely, we need to discuss the remote boundary conditions. The outer boundary of the microscopic problem is connected to the strain gradient plasticity zone of the macroscopic fracture problem. Naturally, the solutions of the strain gradient plasticity zone of the macroscopic fracture problem will be considered as the outer boundary conditions. The continuum solutions discussed above (a $K^{\prime}$ field) which is obtained based on the strain gradient theory as shown in Figs. 4(a) and (b), will be imposed on the outer boundary. The key problem here is how to properly select the radius of the outer boundary, $t$. The $t$ value will be determined through bridging the continuum result with the micro-scale analysis result after the discrete dislocation analysis. In order to analyze the crack growth behavior at micro-scale which influenced by the discrete dislocations, as usual, two typical kinds of the discrete dislocation arrangements will be considered, as shown in Figs. 6(a) and (b) which correspond to plane strain mode I and mode II cases, respectively. The possibility of putting the greatest numbers of dislocations within the region $0<r<t$, will be investigated (corresponding to the limit equilibrium state for each dislocation), where $r$ is the polar coordinate. The arrangement of the discrete dislocations is according to the dislocation equilibrium status: $-1 \leqslant f_{\mathrm{d}} / f_{\mathrm{d}}^{\mathrm{c}} \leqslant 1$, where $f_{\mathrm{d}}$ is dislocation force, $f_{\mathrm{d}}^{\mathrm{c}}=\sigma_{\mathrm{f}} b$ is referred to as the lattice frictional resistance, $\sigma_{\mathrm{f}}$ and $b$ are the critical shear strength along the slip plane and Burgers vector, respectively. When $f_{\mathrm{d}}=f_{\mathrm{d}}^{\mathrm{c}}$, a dislocation is in the limit equilibrium state. The limit equilibrium state will be considered in the present research. Thus, the finite element method can 


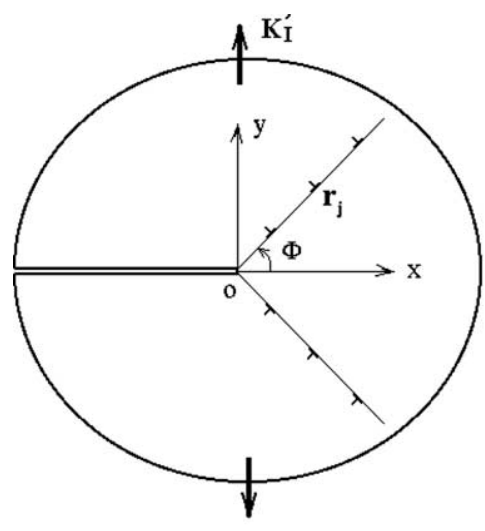

(a) Mode I case

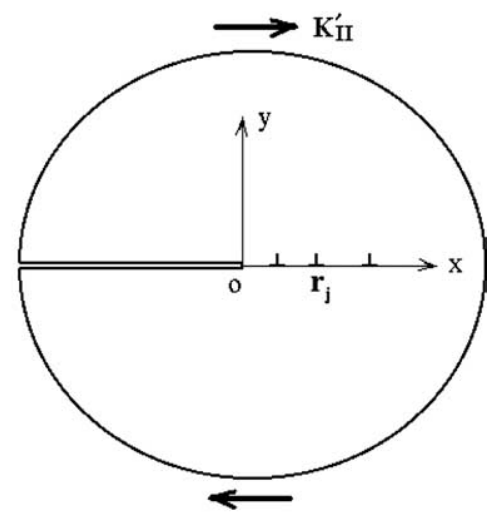

(b) Mode II case

Fig. 6. Discrete distributed dislocation models for micro-scale problems.

be used to compute the interaction of crack with discrete dislocations. The shielding effect on crack growth by the discrete dislocations will be investigated. The dislocation force can be calculated. It is worth noting that Lin and Thomson (1986) obtained the dislocation force formulas (see Appendix B) and the dislocation shielding effect formulations (see Appendix C) in complex form, so one can directly rely the Lin and Thomson's formulas to analyze the interaction of a crack with discrete dislocations. In the present research, we shall adopt the Lin and Thomson's formulas.

Firstly, we investigate the possibility of putting the greatest numbers of discrete dislocations within the submicron region near the crack tip for a stationary crack or for a growing crack. The analytical models we adopt are shown in Figs. 6(a) and (b), respectively, for mode I and mode II. Secondly, the dislocation shielding effects on the microscopic fracture behavior will be studied in detail.

\subsection{Mode I crack case}

The dislocation distribution along a single slip plane will be considered first. Secondly, both the dislocation limit equilibrium location and the possible dislocation number will be determined. For simplicity, we consider the case with one dislocation first, and determine the limit equilibrium location. Lin and Thomson (1986) solved the problem of the crack interaction with the dislocation and formulated the dislocation shielding effect and dislocation force. Their basic formulas are reproduced in Appendices B and C. In the present research, based on the Lin and Thomson's formulas the shielding effect and the dislocation limit equilibrium locations will be calculated using the Newton Raphson method (see Appendix D). For the one-dislocation case, the relation of the dislocation force with its limit equilibrium location is shown in Fig. 7. In the figure, the results for two cases are plotted, respectively, one for $E b / G_{0}\left(1-v^{2}\right)=50$ and the other for $E b / G_{0}\left(1-v^{2}\right)=100$. In both cases, the slip plane direction is $\Phi=60^{\circ}$. Note that $G_{0}=K_{0}^{2}\left(1-v^{2}\right) / E$ is the material microscopic 


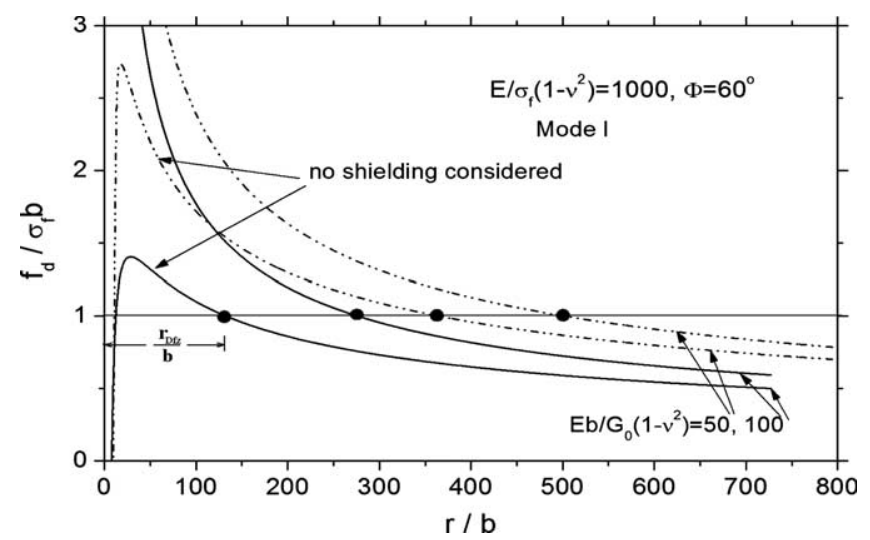

Fig. 7. The location of a single dislocation in limit equilibrium for mode I crack case.

fracture toughness. For each case in Fig. 7, there are two sets of results, corresponding to whether the dislocation shielding effect is considered or not, respectively. There are different limit equilibrium locations and different dislocation-free zone sizes. The dislocation limit equilibrium locations for different cases are indicated in Fig. 7 with solid dots. For the specific material parameters, there exists a peak value on the dislocation force curve for the case without dislocation shielding effect (the case was considered by Lin and Thomson, 1986), however a peak value does not exist when the dislocation shielding effect is considered. A probable equilibrium location for a dislocation is determined through considering the condition when the dislocation force is smaller than the lattice friction force, $\sigma_{\mathrm{f}} b$ in the slip direction. Usually, the dislocation force is taken to be equal to the lattice friction force (the limit equilibrium state). The results shown in Fig. 8 are for $\Phi=30^{\circ}$. Peak values on the dislocation force curves exist for both cases regardless of whether the shielding effect is considered or not. The results shown in Fig. 9 are for the mode II case and exhibit similar features.

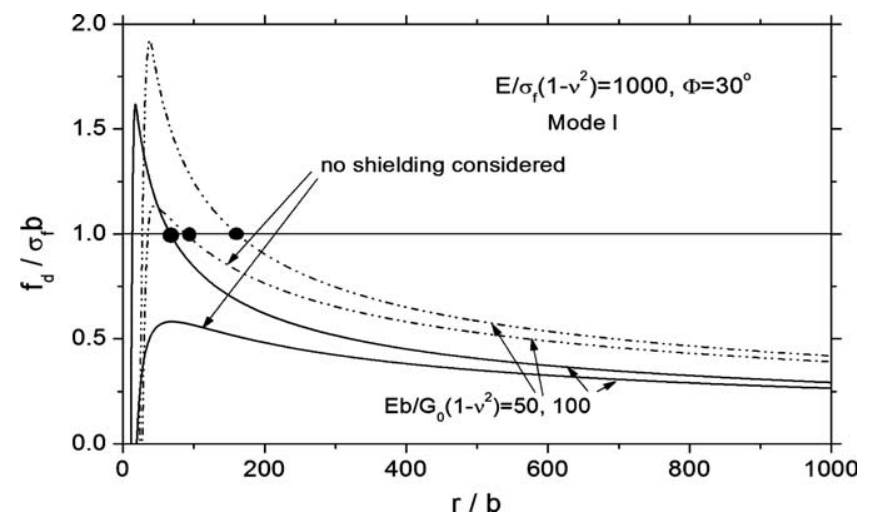

Fig. 8. The location of single dislocation in limit equilibrium for mode I crack case. 


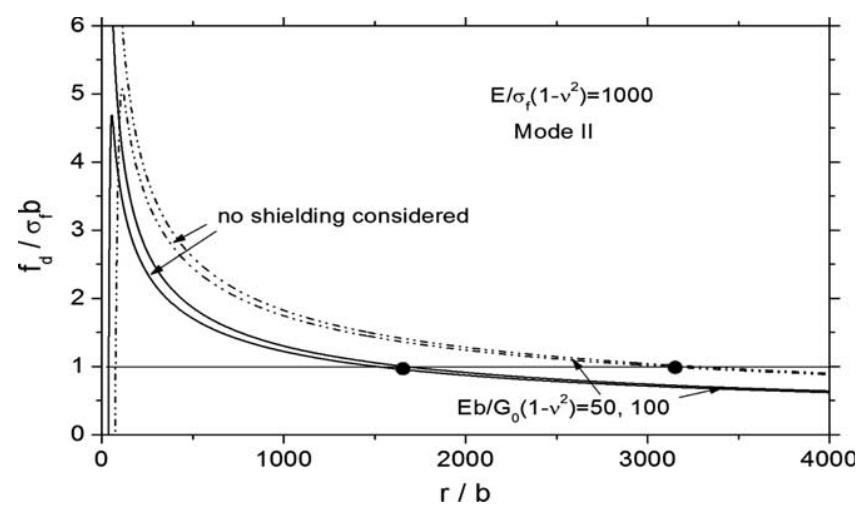

Fig. 9. The location of single dislocation in limit equilibrium for mode II crack case.
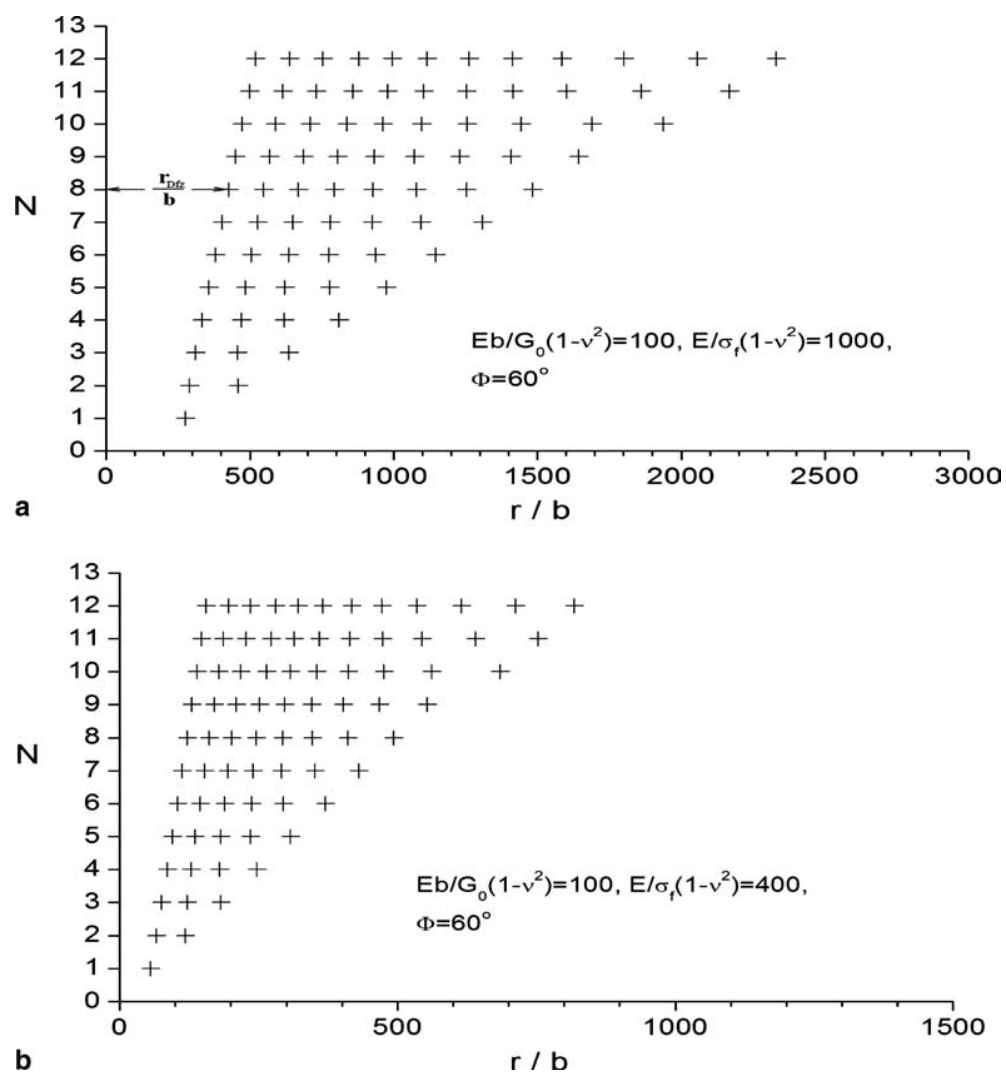

Fig. 10. The numbers of discrete dislocations and the corresponding locations on a slip plane for mode I crack case. 
The limit equilibrium locations when more dislocations are distributed along a single slip plane are investigated here. Figs. 10(a) and (b) show the results corresponding to $E / \sigma_{\mathrm{f}}\left(1-v^{2}\right)=1000$ and 400 , respectively. The other parameters are $E b / G_{0}\left(1-v^{2}\right)=100$ and $\Phi=60^{\circ}$. In Fig. 10(a), different dislocation groups correspond to the different dislocation-free zone sizes. The more the dislocations, the larger the dislocation-free zone size. From Fig. 10(a), when the dislocation number reaches 12 along a slip plane, the discrete dislocation zone extends to the submicron far away from the crack tip $(r \approx 250 \mathrm{~nm}$ for $b \approx 0.1 \mathrm{~nm})$. In Fig. 10(b), as the parameter $E / \sigma_{\mathrm{f}}\left(1-v^{2}\right)$ decreases, the dislocation-free zone size $r_{\text {Dfz }}$ decreases, and the dislocation density increases. The variations of the normalized dislocationfree zone size with the distributed dislocation number are plotted in Fig. 11 for several slip angles. Here, the dislocation-free zone size shows sensitivity to slip plane directions and increases with increasing dislocation number when the slip plane angle is roughly larger than 50 degree. Otherwise, it decreases with increasing dislocation number. Fig. 12 shows the relations between the normalized dislocation-free zone

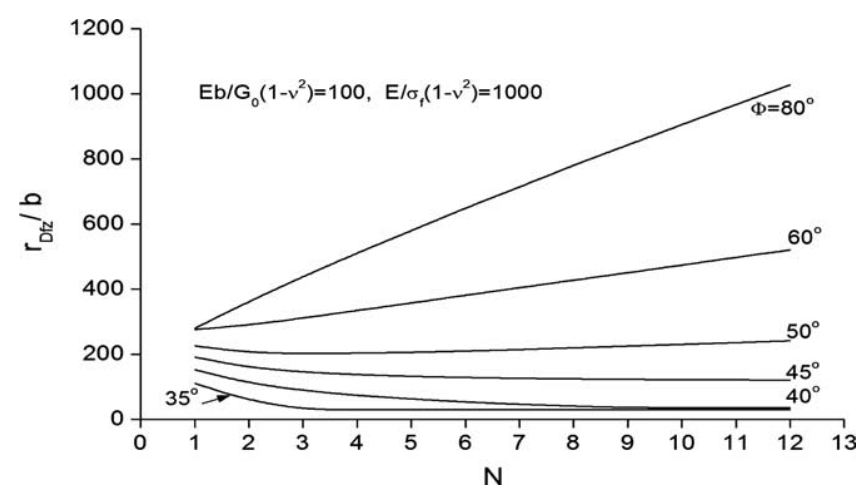

Fig. 11. Relations of the dislocation-free zone size with dislocation numbers for several slip plane directions.

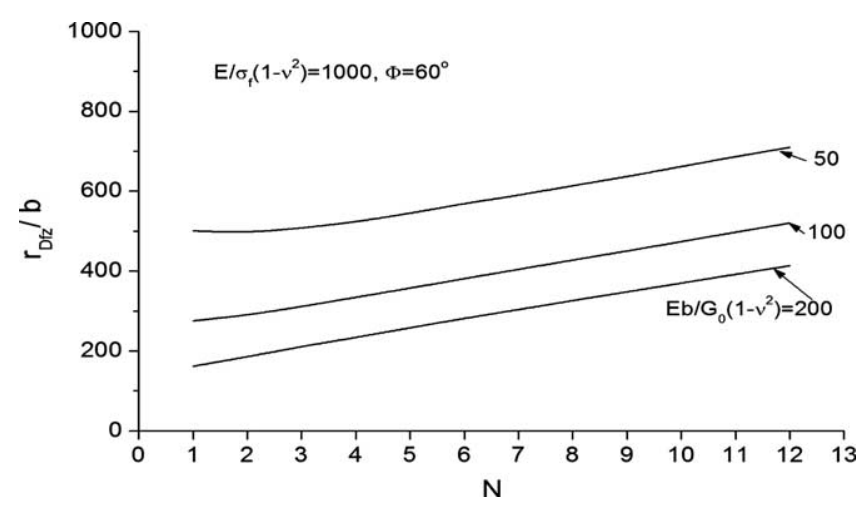

Fig. 12. Relations of the dislocation-free zone size with dislocation numbers for several composition parameter values. 


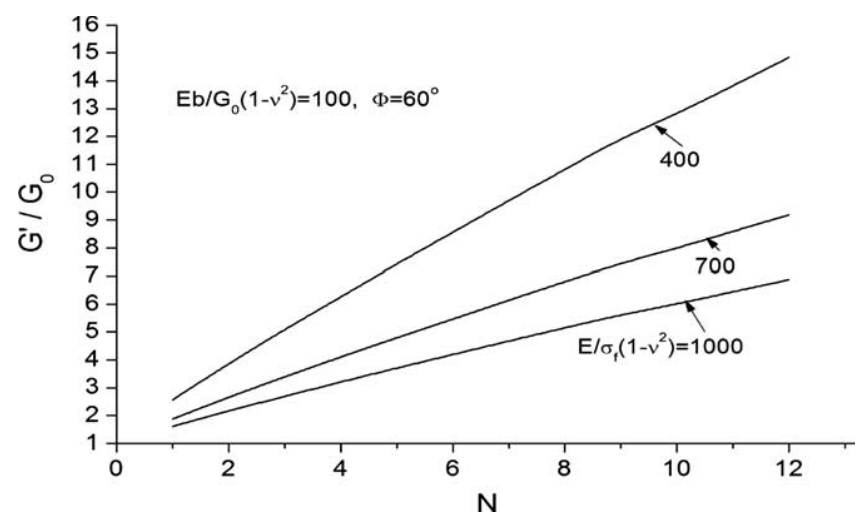

Fig. 13. Variations of energy release rate with dislocation numbers. Dislocation shielding contribution on the crack tip fracture energy.

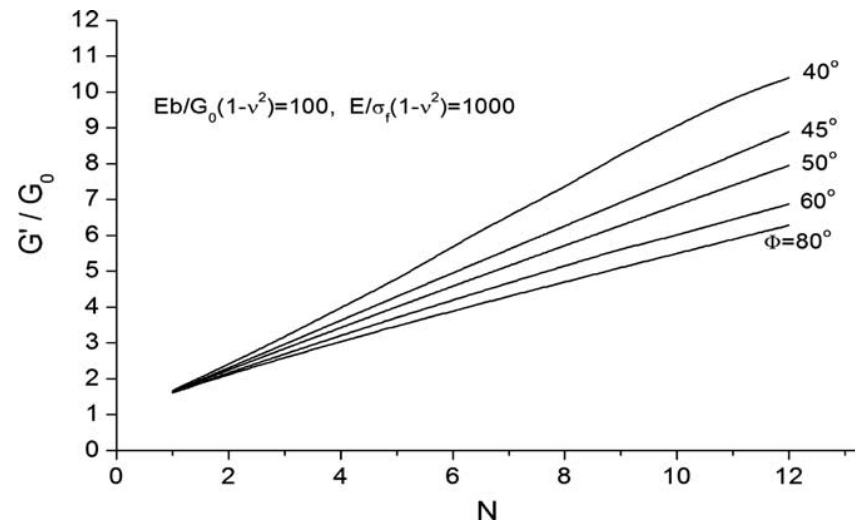

Fig. 14. Variations of energy release rate with dislocation numbers for several slip plane directions.

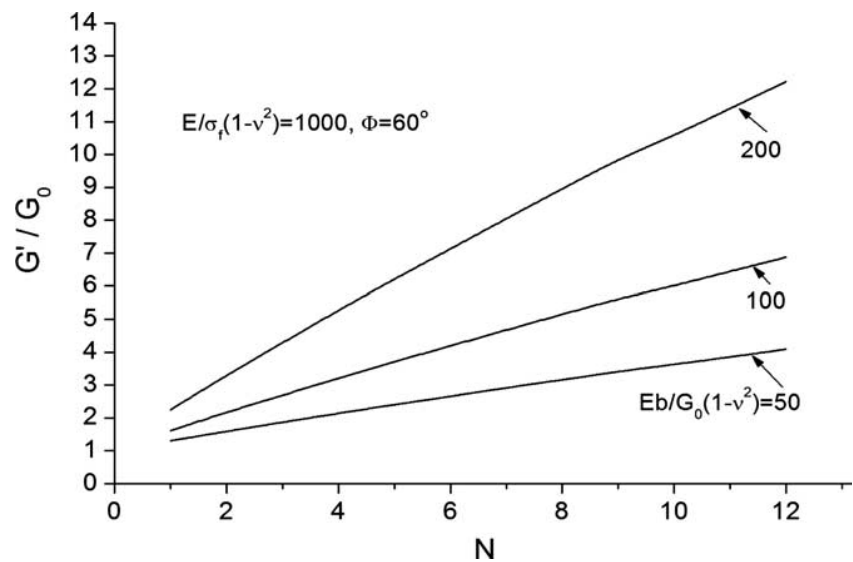

Fig. 15. Variations of energy release rate with dislocation numbers. 
size and the dislocation number for several values of $E b / G_{0}\left(1-v^{2}\right)$. As figure shows, the larger the composition parameter, the smaller the dislocation-free zone size. In other words, the smaller the fracture toughness $G_{0}$, the smaller the dislocation-free zone size. Figs. 13-15 show the dislocation shielding effects on the crack for different parameter values. Here $G^{\prime}=K^{\prime 2}\left(1-v^{2}\right) / E$ is applied energy release rate on the boundary of the microscopic fracture problem. Figs. 13-15 show that the shielding effects increase with increasing dislocation number. Fig. 13 shows that the shielding effects also increase with decreasing value of $E / \sigma_{\mathrm{f}}\left(1-v^{2}\right)$, with increasing slip plane angle (from Fig. 14) and with increasing value of parameter $E b / G_{0}\left(1-v^{2}\right)$ (from Fig. 15).

\subsection{Mode II crack case}

For mode II crack case, the dislocation distribution is along the symmetric line ahead of crack tip, as shown in Fig. 6(b). In order to explore the limit equilibrium location of dislocations, let us discuss the one-dislocation case first. The results in Fig. 9 concern cases where the dislocation shielding effect is considered and is not considered. The equilibrium locations for both cases are very near each other. For the case with more dislocations, the dislocation-free zone sizes decreases as the dislocation number increases, as shown in Figs. 16(a) and (b). This trend is opposite to that for mode I case as seen in Figs. 10(a) and (b). The variations of the normalized dislocation-free zone sizes with dislocation number are plotted in Fig. 17 for several material parameters. With increasing $E b / G_{0}\left(1-v^{2}\right)$, or with decreasing material fracture toughness $G_{0}$, the dislocation-free zone size decreases. This trend is similar to that in the mode I case. The shielding effects in mode II case are plotted in Figs. 18 and 19 for several parameter sets. The influences of the composite parameters $E / \sigma_{\mathrm{f}}\left(1-v^{2}\right)$ and $E b / G_{0}\left(1-v^{2}\right)$ on the shielding effects are similar to that in mode I case. A comparison of Figs. 18, 19 and Fig. 13-15 clearly shows that the shielding effects in mode I case are stronger than those in mode II case.

\section{Steady-state crack growth for mode $I$ in microscopic fracture}

\subsection{The conditions of steady-state crack growth}

The macroscopic fracture solutions for steady-state crack growth have been analyzed and obtained in Section 3. Here we shall focus our attention on the microscopic fracture analysis. We have considered the possibility of discrete dislocations existing along a slip plane within the submicron scale in above sections. Let us further investigate the possibility of dislocation being distributed as crack grows under the steadystate condition, i.e. investigate whether the dislocation pattern can be kept around the crack surface, accompanied by the new slip plane produced near the crack tip. If the crack grows under steady-state conditions, the model for the dislocation pattern is shown in Fig. 20. Here we assume that the space between slip planes is equal to $L$. A question exists as to what is the size of the slip plane space $L$ ? According to 

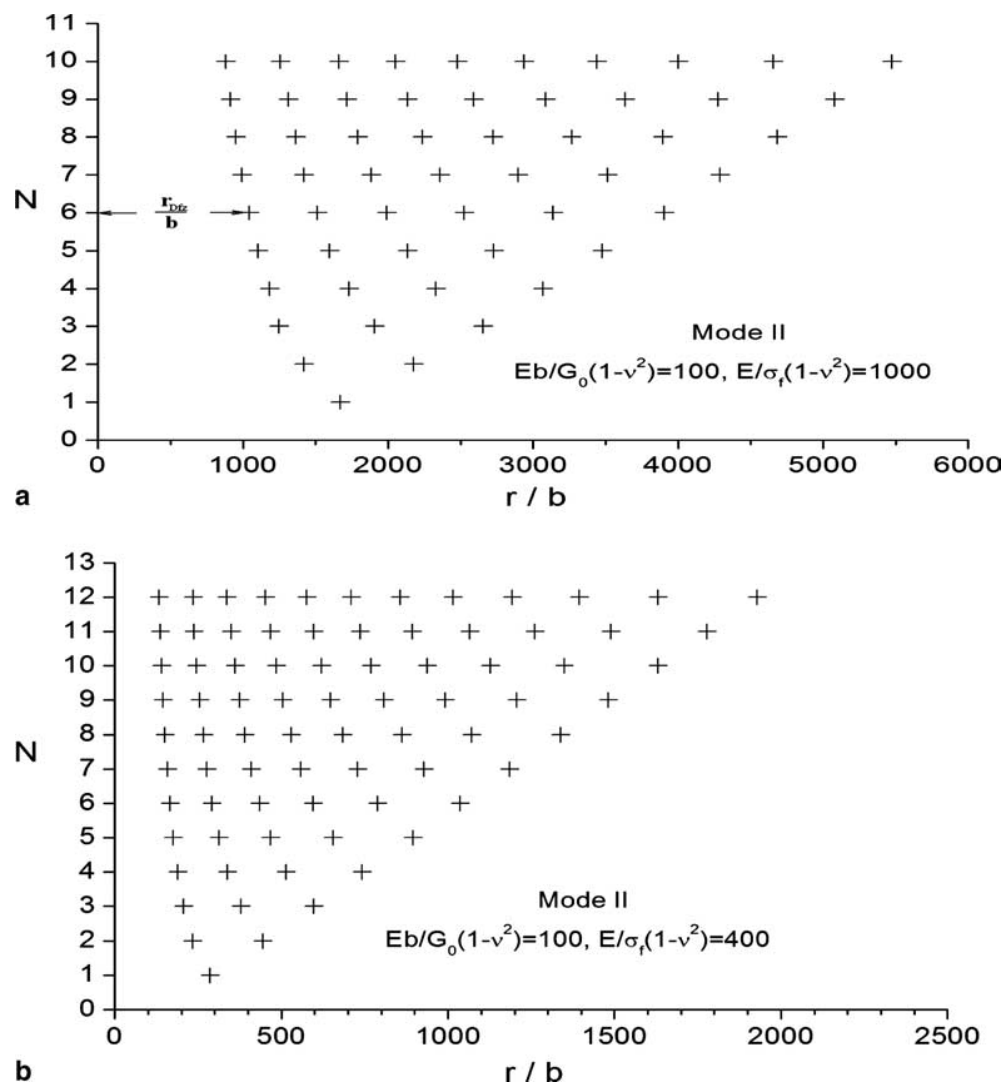

Fig. 16. The numbers of discrete dislocations and the corresponding locations on a slip plane for mode II crack case.

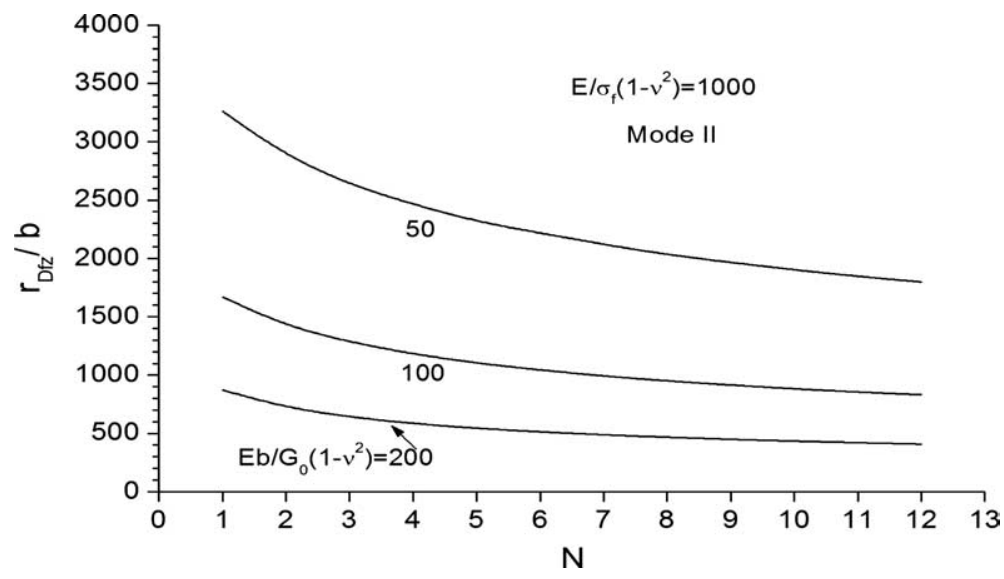

Fig. 17. Relations of the dislocation-free zone size with dislocation numbers for several composition parameter values. 


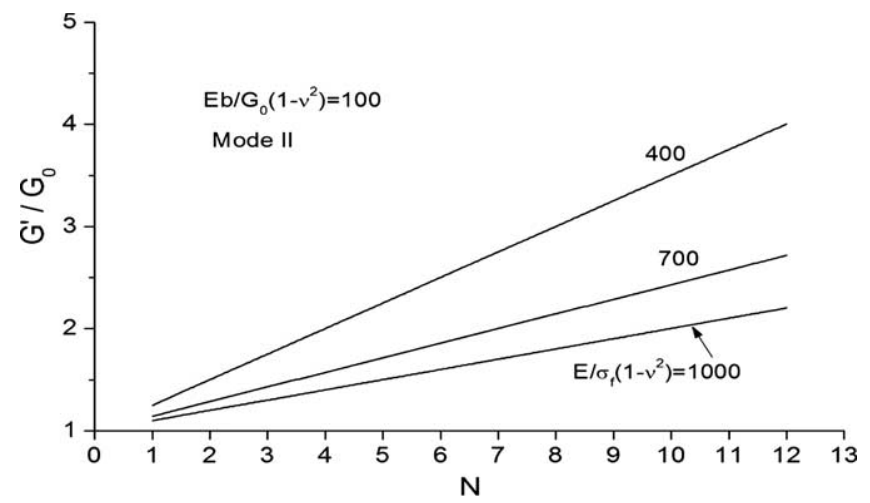

Fig. 18. Variations of energy release rate with dislocation numbers. Dislocation shielding contribution on the crack tip fracture energy (mode II case).

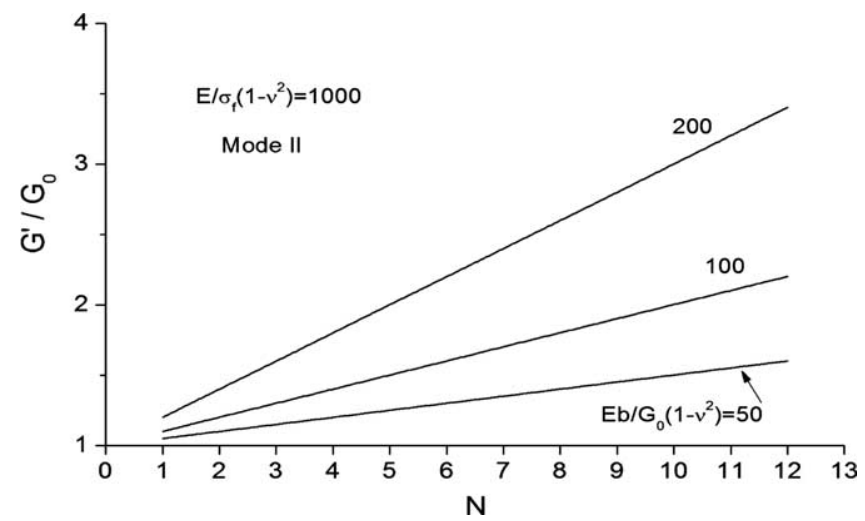

Fig. 19. Variations of energy release rate with dislocation numbers. Dislocation shielding contribution on the crack tip fracture energy (mode II case).

the experimental observation and measurement by Mao and Evans (1997), $L$ is about 1 micron. By calculating the forces for each dislocation or the dislocation pattern, one can examine the stability of the dislocation pattern, i.e., check whether or not $\left|f_{\mathrm{d}} / f_{\mathrm{d}}^{\mathrm{c}}\right| \leqslant 1$ is met as crack grows. Figs. 21-24 show the distributions of the dislocation forces near crack surface as crack grows under steady-state conditions for several composite parameter values. In the figures, four dislocations on each slip surface are considered. On the slip plane near the crack tip, the dislocations are marked with the numbers $1,2,3$ and 4 , according to their distances to the crack tip, from near to far. Figs. 21-24 show the distributions of dislocation forces on each dislocation line (each line is parallel to crack surface). The results shown in Figs. 2123 are for the cases of three slip surface spacings or $L / b=600,300$ and 100 , respectively. The other parameters are taken as $\Phi=60^{\circ}, E b / G_{0}\left(1-v^{2}\right)=100$ and $E / \sigma_{\mathrm{f}}\left(1-v^{2}\right)=1000$. In Figs. 21 and 22 for the cases of $L / b=600$ and 300, the 


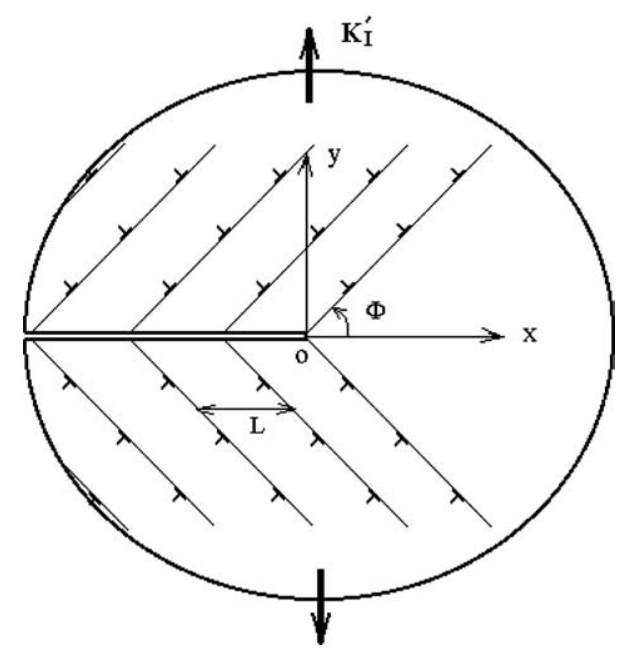

Fig. 20. Multi-slip plane model for the case of steady-state crack growth in the mode I case.

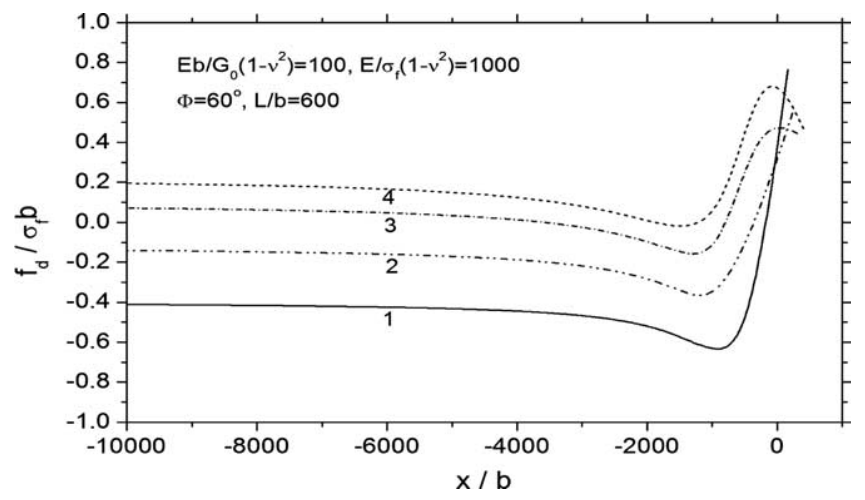

Fig. 21. Dislocation force distributions on area near crack surface for the case when four dislocations are distributed on each slip plane.

dislocation forces in corresponding dislocation patterns are smaller than the critical value. Therefore, both patterns are stable during crack growing. Near the crack tip, the force changes from positive to negative, and it has a big value. At the location far behind the crack tip, the dislocation force is small. In Fig. 23, when the slip plane space $L$ takes on a small value such as $100 b$, the dislocation force near the crack tip is larger than the critical value, and the corresponding dislocation pattern is unstable. For the purpose of investigating the effect of composition parameter $E b / G_{0}\left(1-v^{2}\right)$ and comparing with the results shown in Fig. 21, Fig. 24 plots the results for the case of $E b / G_{0}\left(1-v^{2}\right)=50$. The features of the dislocation force distributions are similar to those for the case of $E b / G_{0}\left(1-v^{2}\right)=100$ shown in Fig. 21. As crack grows step by step, the variations of the shielding effects (energy release rates) 


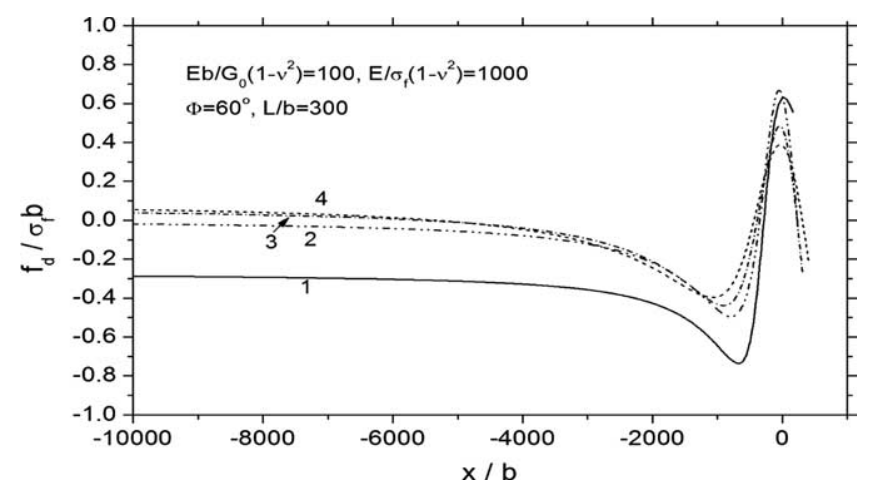

Fig. 22. Dislocation force distributions on area near crack surface for the case when four dislocations are distributed on each slip plane.

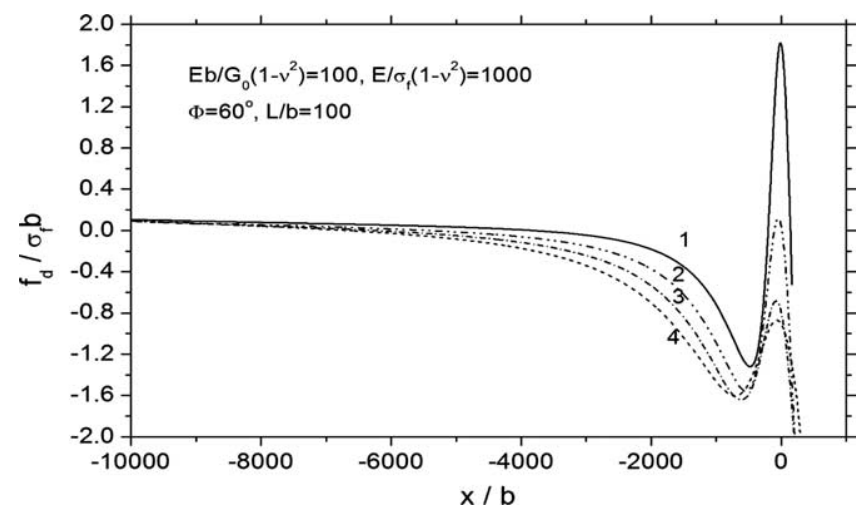

Fig. 23. Dislocation force distributions on area near crack surface for the case when four dislocations are distributed on each slip plane.

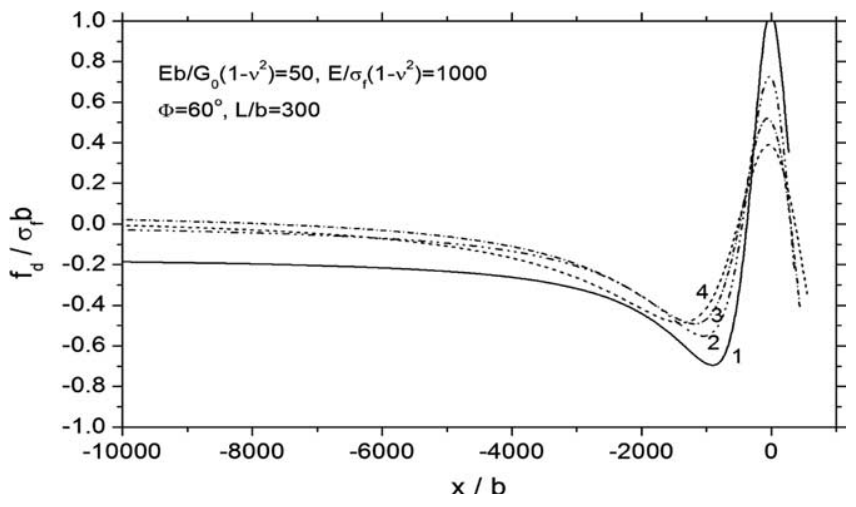

Fig. 24. Dislocation force distributions on area near crack surface for the case when four dislocations are distributed on each slip plane. 
are plotted in Fig. 25 for several material parameters and model parameters. In Fig. 25 , when crack grows for 3-4 times the dislocation-free zone size, the steady-state crack growth condition is approached. Fig. 25 also shows the variations of the normalized energy release rates as a function of the distance of crack growth for several slip plane spacing values. The smaller the slip plane spacing, the larger the normalized energy release rate.

\subsection{The selection of "elastic core" size}

We can directly calculate the energy release rate with the selection of the "elastic core" size $t$ for the steady-state crack growth. By increasing the discrete dislocation number along a slip plane, we can increase corresponding "elastic core" size. Fig. 26

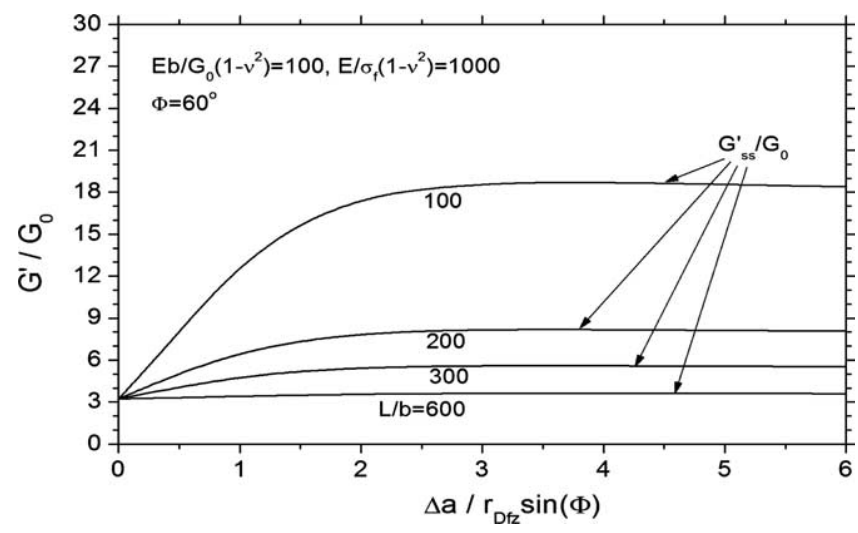

Fig. 25. Variations of energy release rate as crack grows. Steady-state crack growth is easily realized.

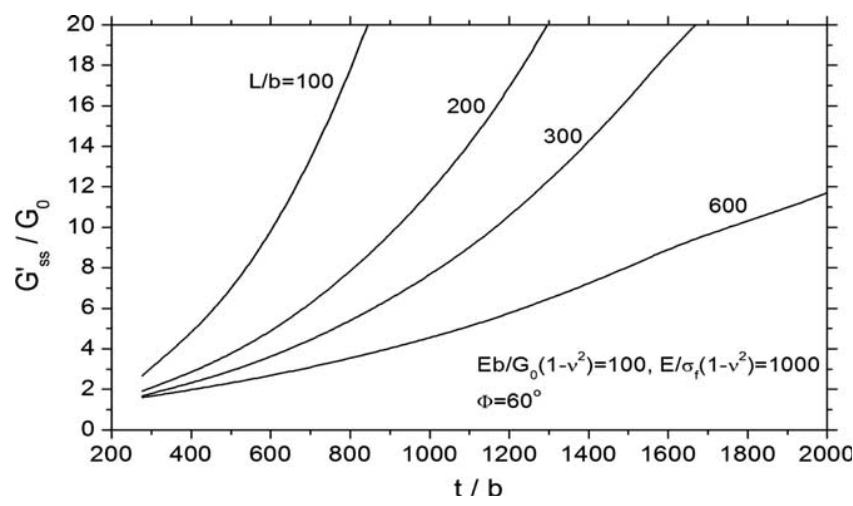

Fig. 26. Variation of energy release rate with elastic core size and space between slip planes under steadystate crack growth condition. 
shows the variations of the normalized steady-state energy release rate with increasing "elastic core" size for several slip plane space values. The toughness ratio from the microscopic theory increases with the "elastic core" size. However, this trend is opposite to what is predicted by the macroscopic theory (from Fig. 3). In order to conveniently compare both microscopic and macroscopic cases, the results shown in Fig. 26 are replotted in Fig. 27 by adopting the same coordinate as that in Fig. 3 for $R_{0} / b=5000$. Simultaneously, the results in Fig. 3 are replotted in Fig. 27. Comparing the results of both microscopic and macroscopic analyses, we can see that both sets of results are sensitive to the selection of the "elastic core" size $t$. If the selected $t$ value is too small, the $G_{\mathrm{ss}} / G_{0}^{\prime}$ value obtained from macroscopic theory will be too large. Similarly, if the selected $t$ is too large, the $G_{\mathrm{ss}}^{\prime} / G_{0}$ value obtained from microscopic theory will be too large. A proper selection of the "elastic core" size $t$

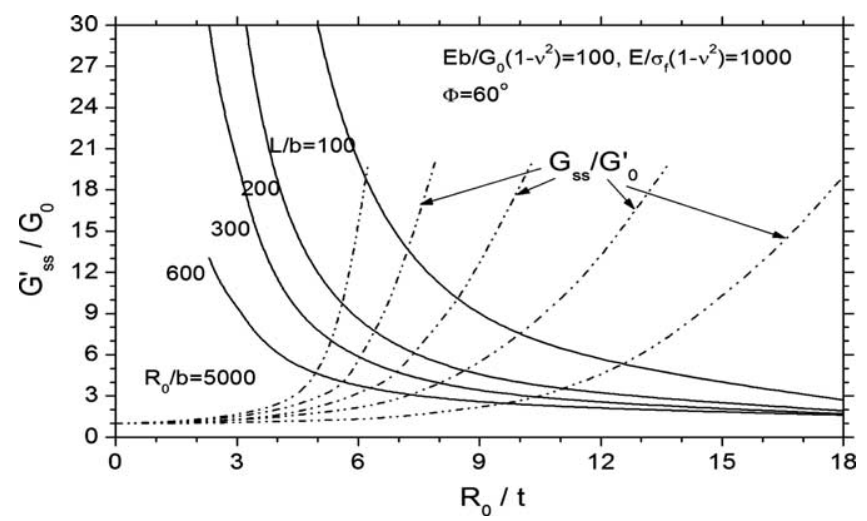

Fig. 27. Comparison of microscopic analysis results with macroscopic analysis results for energy release rate under the steady-state crack growth condition.

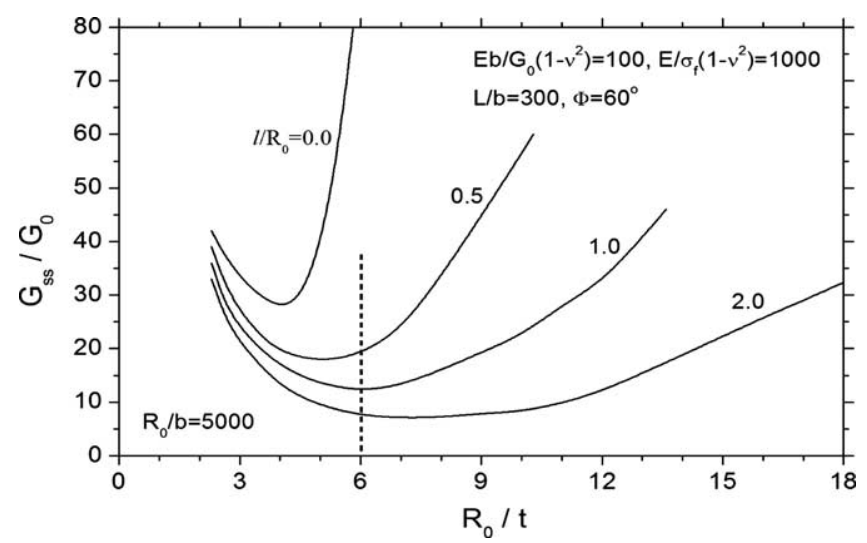

Fig. 28. The determination of elastic core size $t$. From results shown in figure, $R_{0} / t \sim 6$. 
should be such that both theories are relatively insensitive to the selected $t$ value. For seeking the proper selection of $t$, let us examine the following relation for the total energy release rate normalized by the crack tip fracture toughness

$$
\frac{G_{\mathrm{ss}}}{G_{0}}=\frac{G_{\mathrm{ss}}}{G_{\mathrm{ss}}^{\prime}} \frac{G_{\mathrm{ss}}^{\prime}}{G_{0}}=\frac{G_{\mathrm{ss}}}{G_{0}^{\prime}} \frac{G_{\mathrm{ss}}^{\prime}}{G_{0}},
$$

where $G_{0}^{\prime}=G_{\mathrm{ss}}^{\prime}$ from present analysis. Fig. 28 shows the normalized total energy release rate as a function of the "elastic core" size for three values of $R_{0} / b$. The curves in Fig. 28 come from both the microscopic analysis results and the macroscopic analysis results shown in Fig. 27 by using formula (6). Around the value of $R_{0} / t$ approx 6 , the variation of the total energy release rate is insensitive to the selection of the value for $t$, so the proper selection of the elastic core size is about $t \approx R_{0} / 6$, for conventional metallic materials, $R_{0} \approx 1 \mu \mathrm{m}$, so the elastic core size is around hundred nanometers.

\section{Concluding remarks}

For a complete fracture process, two different scale fracture processes are included, i.e., macroscopic fracture and microscopic fracture. In the present research, the complete fracture processes have been studied. The macroscopic fracture process has been analyzed based on the strain gradient plasticity theory. The fracture characteristics, such as the crack tip field, crack tip fracture toughness, as well as the plastic shielding effect, etc. have been investigated. Within the micron scale, the microscopic fracture process has been analyzed based on the discrete dislocation theory and the bridging model. In the bridging model, the macroscopic fracture solution (crack tip field) is taken as the outer boundary condition of the microscopic fracture problem based on the conclusion that the crack tip field of the macroscopic fracture problem is with $1 / 2$ singularity, but with a different magnitude. The shielding effect of discrete dislocations on crack growth has been considered. The selection of the elastic core size $t$, the intersection radius between macro-scale and micro-scale, is determined from the requirement that the total energy release rate be insensitive to the value of $t$. If the selected $t$ value is too small, the shielding effect from plastic deformation is too large, while the shielding effect from discrete dislocations is too small. If the selected $t$ value is too large, the shielding effect from plastic deformation is too small and the shielding effect from discrete dislocations is too large.

Fig. 3 characterizes the variation of the fracture toughness ratio with the elastic core size for the modified SSV model based on the macroscopic analysis, while Fig. 28 can be interpreted as a modification of the results in Fig. 3 when the numbers of discrete dislocations are included within the "elastic core" region. Fig. 28 shows that the variation of the total fracture toughness ratio with $t$ is complicated. At about $t \approx R_{0} / 6$, the toughness ratio changes smoothly and takes its stationary value. As $t$ increases, the toughness ratio increases due to the strong dislocation shielding effect. As $t$ decreases the toughness ratio increases due to the strong plastic shielding effect. 


\section{Acknowledgments}

Y.W. acknowledges the supports of the National Science Foundation of China through Key Project (No. 10432050), Oversea Co-operation Project (No.10428207), Grant 19925211 and the support of the "Bai Ren" Project of the Chinese Academy of Sciences. G.X. gratefully acknowledges the financial support of the National Science Foundation through NIRT award 0134446 and the Army Research Laboratory through Grant DAAD17-02-P-1024.

\section{Appendix A. MSG strain gradient plasticity flow theory}

The MSG flow theory has been derived by Qiu et al. (2003) and Wei et al. (2004). The results are listed as follows:

$$
\begin{aligned}
\dot{\sigma}_{i j}= & K \dot{\varepsilon}_{k k} \delta_{i j}+2 \mu\left(\dot{\varepsilon}_{i j}^{\prime}-\alpha^{\prime} \frac{3 \sigma^{\prime i j}}{2 \sigma} \dot{\varepsilon}^{\mathrm{p}}\right), \\
\dot{\tau}_{i j k}= & c\left[\dot{\eta}_{i j k}+\frac{1}{2}\left(\dot{\eta}_{k i j}+\dot{\eta}_{k j i}\right)+\left(\frac{2 K}{\mu}-\frac{4}{3}\right) \dot{\eta}_{i j k}^{H}\right]-\alpha^{\prime}\left\{\frac{c}{1+a} \frac{3 \sigma_{m n}^{\prime}}{2 \sigma^{2}}\left(\sigma_{k i}^{\prime} \dot{\eta}_{j m n}+\sigma_{k j}^{\prime} \dot{\eta}_{i m n}\right)\right. \\
& +\frac{1}{1+a} \frac{3}{4 \sigma^{2}} G_{i j k m n} \dot{\sigma}_{m n}^{\prime}-\left(\frac{e}{1+a}+\frac{3 \mu}{\sigma}\right) \frac{3}{4 \sigma^{2}} G_{i j k m n} \sigma_{m n}^{\prime} \dot{\varepsilon}^{\mathrm{p}} \\
& \left.+\frac{3 \mu}{\sigma}\left(\tau_{i j k}-\frac{K l_{\varepsilon}^{2}}{6} \eta_{i j k}^{H}\right) \dot{\varepsilon}^{\mathrm{p}}\right\}, \\
\dot{\varepsilon}^{\mathrm{p}}= & \frac{1}{1+a} \frac{1}{\sigma}\left(\sigma_{i j}^{\prime} \dot{\varepsilon}_{i j}-\alpha^{2} \mu b \frac{3}{4 \eta} \eta_{i j k}^{\prime} \dot{\eta}_{i j k}\right),
\end{aligned}
$$

where the flow criterion is considered as (Gao et al., 1999; Huang et al., 2000)

$$
\sigma_{\mathrm{e}}=\sigma=\sqrt{\sigma_{0}^{2} f_{\mathrm{p}}^{2}\left(\varepsilon^{\mathrm{p}}\right)+18 \alpha^{2} \mu^{2} b \eta}=\sigma_{0} \sqrt{f_{\mathrm{p}}^{2}\left(\varepsilon^{\mathrm{p}}\right)+l \eta}
$$

and

$$
\alpha^{\prime}=0 \quad \text { if } \sigma_{\mathrm{e}}<\sigma \text { or } \sigma_{\mathrm{e}}=\sigma \text { and } \dot{\varepsilon}^{\mathrm{p}}<0 ; \quad \alpha^{\prime}=1 \quad \text { if } \sigma_{\mathrm{e}}=\sigma \text { and } \dot{\varepsilon}^{\mathrm{p}} \geqslant 0 .
$$

The parameters and variables in the above relations are defined as

$$
\begin{aligned}
& c=\frac{\mu l_{\varepsilon}^{2}}{12}, \quad a=\frac{\sigma_{0}^{2}}{3 \mu \sigma} f_{\mathrm{p}} f_{\mathrm{p}}^{\prime}, \quad e=\frac{f_{\mathrm{p}}^{\prime}}{f_{\mathrm{p}}}+\frac{f_{\mathrm{p}}^{\prime \prime}}{f_{\mathrm{p}}^{\prime}}, \\
& G_{i j k m n}=\sigma_{k i}^{\prime}\left(\tau_{j m n}+\tau_{j n m}-\tau_{m n j}\right)+\sigma_{k j}^{\prime}\left(\tau_{i m n}+\tau_{i n m}-\tau_{m n i}\right),
\end{aligned}
$$

where $f_{\mathrm{p}}\left(\varepsilon^{\mathrm{p}}\right)$ is the function in the uniaxial stress-plastic strain relation determined from uniaxial tension:

$$
\sigma_{\mathrm{I}}=\sigma_{0} f_{\mathrm{p}}\left(\varepsilon^{\mathrm{p}}\right),
$$


$\sigma_{0}$ is a reference stress; $\varepsilon^{\mathrm{p}}$ is accumulative plastic strain; $b$ is the Burgers vector; $\alpha$ is an empirical coefficient in the Taylor model and is taken the value around 0.3 for the conventional metals; $K=E / 3(1-2 v)$ and $\mu=E / 2(1+v)$ are the elastic bulk modulus and shear modulus, respectively; $l_{\varepsilon}$ is the cell size, and from analysis of Gao et al. (1999), Huang et al. (2000, 2004) and Qiu et al. (2003),

$$
l_{\varepsilon}=10 \mu b / \sigma_{\mathrm{Y}}, \quad l=18 \alpha^{2}\left(\frac{\mu}{\sigma_{0}}\right)^{2} b,
$$

$l$ is the length scale parameter characterizing a strength of the strain gradient or the geometrically-necessary dislocation (Gao et al., 1999; Huang et al., 2000; Shi et al., 2004), according to the research for the MSG theory in Gao et al. (1999), the value of the length parameter $l$ falls the region of 1-10 microns for the typical metal materials; $\eta$ is the effective strain gradient and can be expressed by the components of strain gradient $\eta_{i j k}=u_{k, i j}$ as

$$
\eta=\sqrt{\frac{1}{4} \eta_{i j k}^{\prime} \eta_{i j k}^{\prime}}
$$

and the deviatoric and volumetric components of $\eta_{i j k}$ are dictated as (Fleck and Hutchinson, 1997)

$$
\eta_{i j k}^{\prime}=\eta_{i j k}-\eta_{i j k}^{H}, \quad \eta_{i j k}^{H}=\frac{1}{4}\left(\delta_{i k} \eta_{j p p}+\delta_{j k} \eta_{i p p}\right) .
$$

A piecewise power law hardening stress-strain relation is considered to characterize the solid behaving at macro-scale,

$$
\sigma_{\mathrm{I}}= \begin{cases}E \varepsilon & \text { for } \varepsilon \leqslant \frac{\sigma_{\mathrm{Y}}}{E} \\ \sigma_{0} \varepsilon^{N} & \text { for } \varepsilon \geqslant \frac{\sigma_{\mathrm{Y}}}{E}\end{cases}
$$

where $N$ is material strain hardening exponent. From (A.7) and (A.11), one has

$$
\sigma_{0}=\sigma_{\mathrm{Y}}\left(E / \sigma_{\mathrm{Y}}\right)^{N} \text {. }
$$

\section{Appendix B. Dislocation force formulas from Lin and Thomson (1986)}

$$
\begin{aligned}
f_{\mathrm{d}}= & f_{k \mathrm{~d}}+f_{\mathrm{d}^{2}}+\sum_{j} f_{\mathrm{dd}_{j}}+f_{\mathrm{ext}} \\
\bar{f}_{k \mathrm{~d}}^{(K)}= & \frac{K_{\mathrm{III}} b_{\mathrm{s}}}{\sqrt{2 \pi \varsigma}}+\frac{1}{2 \mathrm{i} \sqrt{2 \pi \varsigma}}\left\{K b_{\mathrm{e}}-\bar{K} b_{\mathrm{e}}+\frac{\bar{K} b_{\mathrm{e}}(\varsigma-\bar{\varsigma})}{2 \varsigma}-\bar{K} \bar{b}_{\mathrm{e}}\right\}-\frac{K \bar{b}_{\mathrm{e}}}{2 \mathrm{i} \sqrt{2 \pi \bar{\varsigma}}} \\
\bar{f}_{\mathrm{d}^{2}}= & -\frac{\mu b_{\mathrm{s}}^{2}}{4 \pi}\left\{\frac{1}{2 \varsigma}+\frac{1}{\varsigma-\bar{\varsigma}}-\sqrt{\frac{\bar{\varsigma}}{\varsigma}} \frac{1}{\varsigma-\bar{\varsigma}}\right\}-\frac{\mu}{4 \pi(1-v)}\left\{\frac{b_{\mathrm{e}}^{2} \sqrt{\bar{\varsigma}}}{4 \varsigma^{3 / 2}}\left(\sqrt{\frac{\bar{\varsigma}}{\varsigma}}+1\right)\right. \\
& \left.+\frac{b_{\mathrm{e}} \bar{b}_{\mathrm{e}}}{4}\left[\frac{3}{\varsigma}+\frac{1}{2 \sqrt{\varsigma \bar{\zeta}}}\left(1-\frac{\bar{\varsigma}}{\varsigma}\right)+\frac{3-\sqrt{\bar{\varsigma} / \varsigma}}{\sqrt{\varsigma}(\sqrt{\varsigma}+\sqrt{\bar{\varsigma}})}\right]-\frac{\bar{b}_{\mathrm{e}}^{2}}{4 \bar{\varsigma}}\left(1+\sqrt{\frac{\bar{\varsigma}}{\varsigma}}\right)\right\}
\end{aligned}
$$




$$
\begin{aligned}
& \bar{f}_{\mathrm{dd}_{j}}=\frac{\mu b_{\mathrm{s}} b_{\mathrm{s}}(j)}{4 \pi}\left\{\frac{1}{\varsigma-\varsigma_{j}}-\frac{1}{\varsigma-\bar{\varsigma}_{j}}+\sqrt{\frac{\varsigma_{j}}{\varsigma}} \frac{1}{\varsigma-\varsigma_{j}}+\sqrt{\frac{\bar{\varsigma}_{j}}{\varsigma}} \frac{1}{\varsigma-\bar{\varsigma}_{j}}\right\} \\
& +\frac{\mu}{8 \pi(1-v)}\left\{\left(b_{\mathrm{e}} b_{\mathrm{e}}(j)+\bar{b}_{\mathrm{e}} b_{\mathrm{e}}(j)+b_{\mathrm{e}} b_{\mathrm{e}}(j)\right)\left[\frac{1}{\xi_{j}}\left(\sqrt{\frac{\varsigma_{j}}{\varsigma}}+1\right)+\frac{1}{\xi_{j}^{*}}\left(\sqrt{\frac{\bar{\zeta}_{j}}{\varsigma}}-1\right)\right] c\right. \\
& -\bar{b}_{\mathrm{e}} \bar{b}_{\mathrm{e}}(j)\left[\frac{1}{\bar{\xi}_{j}}\left(\sqrt{\frac{\bar{\zeta}_{j}}{\bar{\varsigma}}}+1\right)+\frac{1}{\bar{\xi}_{j}^{*}}\left(\sqrt{\frac{\varsigma_{j}}{\bar{\zeta}}}-1\right)\right]-2 b_{\mathrm{e}} b_{\mathrm{e}}(j) \mathrm{i} y\left[\frac{1}{\xi_{j}^{2}}\left(\sqrt{\frac{\varsigma_{j}}{\varsigma}}+1\right)\right. \\
& \left.+\frac{1}{\xi_{j}^{* 2}}\left(\sqrt{\frac{\bar{\varsigma}_{j}}{\varsigma}}-1\right)\right]+\frac{b_{\mathrm{e}} b_{\mathrm{e}}(j) \mathrm{i} y_{j}}{\xi_{j}^{2}}\left[\sqrt{\frac{\varsigma_{j}}{\varsigma}}+\sqrt{\frac{\varsigma}{\varsigma_{j}}+2}\right]-\left(\frac{\bar{b}_{\mathrm{e}} \bar{b}_{\mathrm{e}}(j)+b_{\mathrm{e}} \bar{b}(j)}{\xi_{j}^{* 2}} \mathrm{i} y_{j}\right. \\
& \left.+\frac{4 b_{\mathrm{e}} \bar{b}_{\mathrm{e}}(j) y y_{j}}{\xi_{j}^{* 3}}\right)\left[\sqrt{\frac{\bar{\varsigma}_{j}}{\varsigma}}+\sqrt{\frac{\varsigma}{\bar{\varsigma}_{j}}-2}\right]-\frac{\bar{b}_{\mathrm{e}} b_{\mathrm{e}}(j) \mathrm{i} y_{j}}{\bar{\xi}_{j}^{* 2}}\left[\sqrt{\frac{\varsigma_{j}}{\bar{\varsigma}}}+\sqrt{\frac{\bar{\varsigma}}{\varsigma_{j}}}-2\right] \\
& \left.-\frac{b_{\mathrm{e}} b_{\mathrm{e}}(j) \mathrm{i} y}{\varsigma^{3 / 2}}\left(\frac{\sqrt{\varsigma_{1}}}{\xi_{j}}+\frac{\sqrt{\bar{\varsigma}_{1}}}{\xi_{j}^{*}}\right)+\frac{b_{\mathrm{e}} \bar{b}_{\mathrm{e}}(j) y y_{j}}{\xi_{j}^{* 2} \varsigma}\left(\sqrt{\frac{\varsigma}{\bar{\varsigma}_{j}}}-\sqrt{\frac{\bar{\varsigma}_{j}}{\varsigma}}\right)\right\}
\end{aligned}
$$

where, $K=K_{\mathrm{I}}+\mathrm{i} K_{\mathrm{II}}$

$$
\xi_{j}=\varsigma-\varsigma_{j}, \quad \xi_{j}^{*}=\varsigma-\bar{\varsigma}_{j}, \quad y=\frac{1}{2 \mathrm{i}}(\varsigma-\bar{\varsigma}), \quad y_{j}=\frac{1}{2 \mathrm{i}}\left(\varsigma_{j}-\bar{\varsigma}_{j}\right)
$$

\section{Appendix C. Shielded crack}

In plane strain elastic fracture $K$-fields which account for effects of discrete dislocations, the dislocation fields contribute to the $K$-field and cause the local (crack tip) stress intensity factor $K_{0}$ to be composed of the applied $K$ and the equivalent $K^{\mathrm{D}}$ from discrete dislocations as (Lin and Thomson, 1986)

$$
K_{0}=K-\sum_{j} K^{\mathrm{D}}(j)
$$

where

$$
\begin{aligned}
& \bar{K}^{\mathrm{D}}(j)=\frac{\mu}{2 \mathrm{i}(1-v)}\left[\frac{b_{\mathrm{e}}(j)}{\sqrt{2 \pi \varsigma_{j}}}+\frac{b_{\mathrm{e}}(j)}{\sqrt{2 \pi \bar{\varsigma}_{j}}}+\frac{\pi \bar{b}_{\mathrm{e}}(j)\left(\varsigma_{j}-\bar{\varsigma}_{j}\right)}{\left(2 \pi \bar{\varsigma}_{j}\right)^{3 / 2}}\right], \\
& K_{0}=K_{0 \mathrm{I}}+\mathrm{i} K_{0 \mathrm{II}}, \quad K^{\mathrm{D}}=K_{\mathrm{I}}^{\mathrm{D}}+\mathrm{i} K_{\mathrm{II}}^{\mathrm{D}}, \quad b_{\mathrm{e}}(j)=b_{1}(j)+\mathrm{i} b_{2}(j),
\end{aligned}
$$

$j=1,2, \ldots, N . N$ is number of total dislocations. 
Energy release rates can be obtained for the following cases:

Mode I case:

$$
G^{\prime}=\frac{K_{\mathrm{I}}^{2}\left(1-v^{2}\right)}{E}=G_{0}\left[1+\frac{1}{K_{0 \mathrm{I}}} \sum_{j} K_{\mathrm{I}}^{\mathrm{D}}(j)\right]^{2},
$$

where

$$
G_{0}=\frac{K_{0 \mathrm{I}}^{2}\left(1-v^{2}\right)}{E} .
$$

Mode II case:

$$
G^{\prime}=\frac{K_{\mathrm{II}}^{2}\left(1-v^{2}\right)}{E}=G_{0}\left[1+\frac{1}{K_{0 \mathrm{II}}} \sum_{j} K_{\mathrm{II}}^{D}(j)\right]^{2},
$$

where

$$
G_{0}=\frac{K_{0 I I}^{2}\left(1-v^{2}\right)}{E} .
$$

\section{Appendix D. Newton-Raphson method for determining the dislocation limit equilibrium locations}

$$
\text { Let } F\left(x_{1}, x_{2}, x_{3}, \ldots, x_{N}\right)=\sum_{i=1, N}\left[f_{d(i)}\left(x_{1}, x_{2}, x_{3}, \ldots, x_{N}\right)-f_{\mathrm{d}}^{\mathrm{c}}\right]^{2} \text {, }
$$

where $\left(x_{1}, x_{2}, x_{3}, \ldots, x_{N}\right)$ are the locations of dislocations. $f_{\mathrm{d}(i)}$ expression has been given in formulas (B.1)-(B.4) in Appendix B. When the right-hand side of (D.1) is equal to zero, $\left(x_{1}, x_{2}, x_{3}, \ldots, x_{N}\right)$ are the equilibrium positions of dislocations. The iterative formula of Newton-Raphson method for finding the dislocation limit equilibrium locations can be obtained from (D.1) as

$$
\begin{aligned}
& \left(x_{1}^{(k+1)} x_{2}^{(k+1)} \ldots x_{N}^{(k+1)}\right)^{T}=\left(x_{1}^{(k)} x_{2}^{(k)} \cdots x_{N}^{(k)}\right)^{\mathrm{T}} \\
& \quad-\frac{F\left(x_{1}^{(k)}, x_{2}^{(k)}, \ldots, x_{N}^{(k)}\right)}{\left(\frac{\partial F}{\partial x_{1}}\right)_{(k)}^{2}+\left(\frac{\partial F}{\partial x_{2}}\right)_{(k)}^{2}+\cdots+\left(\frac{\partial F}{\partial x_{N}}\right)_{(k)}^{2}}\left(\frac{\partial F_{(k)}}{\partial x_{1}} \frac{\partial F_{(k)}}{\partial x_{2}} \cdots \frac{\partial F_{(k)}}{\partial x_{N}}\right)^{\mathrm{T}} .
\end{aligned}
$$

\section{References}

Argon, A.S., 2001. Mechanics and physics of brittle to ductile transitions in fracture. J. Eng. Mater. Technol. 123, 1-11.

Beltz, G.E., Rice, J.R., Shih, C.F., Xia, L., 1996. A self-consistent model for cleavage in the presence of plastic flow. Acta Mater. 44, 3943-3954. 
Beltz, G.E., Wang, J.S., 1992. Crack direction effects along copper sapphire interfaces. Acta Metall. Mater. 40, 1675-1683.

Betegon, C., Hancock, J.W., 1991. Two-parameter characterization of elastic-plastic crack-tip fields. J. Appl. Mech. 113, 104-110.

Bonfoh, N., Lipinski, P., Carmasol, A., Tiem, S., 2004. Micromechanical modeling of ductile damage of polycrystalline materials with heterogeneous particles. Int. J. Plasticity 20, 85-106.

Chen, S.H., Wang, T.C., 2002. Finite element solutions for plane strain mode I crack with strain gradient effects. Int. J. Solids Struct. 39, 1241-1257.

Evans, A.G., Hutchinson, J.W., Wei, Y., 1999. Interface adhesion: effects of plasticity and segregation. Acta Mater. 47, 4093-4113.

Fleck, N.A., Hutchinson, J.W., 1997. Strain gradient plasticity. In: Hutchinson, J.W., Wu, T.Y. (Eds.), Advances in Applied Mechanics, vol. 33. Academic, New York, pp. 295-361.

Gao, H., Huang, Y., Nix, W.D., Hutchinson, J.W., 1999. Mechanism-based strain gradient plasticity -I. Theory. J. Mech. Phys. Solids 47, 1239-1263.

Giessen, V.D.E., Needleman, A., 1995. Discrete dislocation plasticity: simple plannar model. Model. Simul. Sci. Eng. 3, 689-735.

Hong, T., Smith, J.R., Srolovitz, D.J., 1994. Metal-ceramic adhesion - a first principle study of MgO/Al and $\mathrm{MgO} / \mathrm{Ag}$. J. Adhes. Sci. Technol. 8, 837-851.

Hsia, K.J., Suo, Z., Yang, W., 1994. Cleavage due to dislocation confinement in layered materials. J. Mech. Phys. Solids 42, 877-896.

Huang, Y., Gao, H., Nix, W.D., Hutchinson, J.W., 2000. Mechanism-based strain gradient plasticity-II. Anal. J. Mech. Phys. Solids 48, 99-128.

Huang, Y., Qu, S., Hwang, K.C., Li, M., Gao, H., 2004. A conventional theory of mechanism-based strain gradient plasticity. Int. J. Plasticity 20, 753-782.

Jiang, H., Huang, Y., Zhuang, Z., Hwang, K.C., 2001. Fracture in mechanism-based strain gradient plasticity. J. Mech. Phys. Solids 49, 979-993.

Khan, S.M.A, Khraisheh, M.K., 2004. A new criterion for mixed mode fracture initiation based on the crack tip plastic core region. Int. J. Plasticity 20, 55-84.

Lin, I.H., Thomson, R., 1986. Cleavage, dislocation emission, and shielding for cracks under general loading. Acta Metall. 34, 187-206.

Lipkin, D.M., Clarke, D.R., Beltz, G.E., 1996. A strain gradient model of cleavage fracture in plastically deforming materials. Acta Mater. 44, 4051-4058.

Mao, S.X., Evans, A.G., 1997. The influence of blunting on crack growth at oxide/metal interfaces. Acta Mater. 45, 4263-4270.

Mao, S.X., Li, M.Z., 1999. Effects of dislocation shielding on interface crack initiation and growth in metal/ceramic layered materials. J. Mech. Phys. Solids 47, 2351-2379.

Needleman, A., 1987. A continuum model for void nucleation by inclusion debonding. J. Appl. Mech. 54, $525-531$.

O'Dowd, N.P., Shih, C.F., 1991. Family of crack tip fields characterized by a triaxiality parameter-I. Structure of fields. J. Mech. Phys. Solids 39, 989-1015.

O’Dowd, N.P., Stout, M.G., Shih, C.F., 1992. Fracture-toughness of alumina niobium interface experiments and analyses. Philos. Mag. A 66, 1037-1064.

Qiu, X., Huang, Y, Wei, Y., Gao, H., Hwang, K.C., 2003. The flow theory of mechanism-based strain gradient plasticity. Mech. Mater. 35, 245-258.

Raynolds, J.E., Smith, J.R., Zhao, G.L., Srolovitz, D.J., 1996. Adhesion in NiAl-Cr from first principles. Phys. Rev. B 53, 13883-13890.

Reimanis, I.E., Dalgleish, B.J., Evans, A.G., 1991. The fracture-resistance of a model metal ceramic interface. Acta Metall. Mater. 39, 3133-3141.

Rice, J.R., 1992. Dislocation nucleation from a crack tip: an analysis based on the Peierls concept. J. Mech. Phys. Solids 40, 239-271.

Rice, J.R., Thomson, R., 1974. Ductile versus brittle behaviour of crystals. Philos. Mag. 29, 73-97.

Shi, M.X., Huang, Y., Gao, H., 2004. The $J$-integral and geometrically necessary dislocations in nonuniform plastic deformation. Int. J. Plasticity 20, 1739-1762. 
Suo, Z., Shih, C.F., Varias, A.G., 1993. A theory for cleavage cracking in the presence of plastic flow. Acta Metall. Mater. 41, 151-1557.

Tvergaard, V., 1997. Cleavage crack growth resistance due to plastic flow around a near-tip dislocationfree region. J. Mech. Phys. Solids 45, 1007-1023.

Tvergaard, V., Hutchinson, J.W., 1993. The influence of plasticity on mixed mode interface toughness. J. Mech. Phys. Solids 41, 1119-1135.

Wang, T.C., 1998. Dislocation behaviours ahead of crack tip. Int. J. Solids Struct. 35, 5033-5050.

Wei, Y., Wang, T.C., 1995a. Fracture criterion based on the higher-order asymptotic fields. Int. J. Fract. $73,39-50$.

Wei, Y., Wang, T.C., 1995b. Characterization of elastic-plastic fields near stationary crack tip and fracture criterion. Engin. Fract. Mech. 51, 547-553.

Wei, Y., Hutchinson, J.W., 1997a. Steady-state crack growth and work of fracture for solids characterized by strain gradient plasticity. J. Mech. Phys. Solids 45, 1253-1273.

Wei, Y., Hutchinson, J.W., 1997b. Nonlinear delamination mechanics for thin films. J. Mech. Phys. Solids $45,1137-1159$.

Wei, Y., Hutchinson, J.W., 1999. Models of interface separation accompanied by plastic dissipation at multiple scales. Int. J. Fract. 95, 1-17.

Wei, Y., Qiu, X., Hwang, K.C., 2004. Steady-state crack growth and fracture work based on the theory of mechanism-based strain gradient plasticity. Eng. Fract. Mech. 71, 107-125.

Xia, L., Wang, T.C., Shih, C.F., 1993. Higher-order analysis of crack tip fields in elastic power-law hardening materials. J. Mech. Phys. Solids 41, 665-687.

Xin, Y.B., Hsia, K.J., 1997. Simulation of the brittle-ductile transition in silicon single crystals using dislocation mechanics. Acta Mater. 45, 1747-1759.

Xu, G., Argon, A.S., Ortiz, M., 1995. Nucleation of dislocations from crack tips under mixed modes of loading: implications for brittle against ductile behaviour of crystals. Philos. Mag. A 72, 415-451.

$\mathrm{Xu}, \mathrm{G}$., Argon, A.S., 1997. Critical configurations for dislocation nucleation from crack tips. Philos. Mag. A $75,341-367$.

Yang, W., Tang, J.C., Ing, Y.S., Ma, C.C., 2001. Transient dislocation emission from a crack tip. J. Mech. Phys. Solids 49, 2431-2453. 